\section{SAT0522 COMPARISON OF CHILDREN CARRYING E148Q VARIANT WITH CHILDREN CARRYING HOMOZYGOUS PATHOGENIC VARIANTS}

Ayşe Tanatar, Şerife Gül Karadağ, Hafize Emine Sonmez, Mustafa Çakan, Nuray Aktay Ayaz. University of Health Science, Kanuni Sultan Süleyman Research and Training Hospital, Pediatric Rheumatology, Istanbul, Turkey

Background: Familial Mediterranean fever (FMF) is the most frequent autoinflammatory disease. Nearly 300 MEFV variants had been reported and recorded in INFEVERS database. The most common disease-associated variants are mapped on exon 2 and 10 of chromosome 16 . Although $E 148 Q$ variant is the most common one among carriers, its role as a disease causing mutation is still debate (1).

Objectives: The aim of our study was to evaluate and compare the demographic data, clinical features and severity scores of patients carrying only E148Q variant with the patients homozygous for pathogenic MEFV mutations (M694V, M694I, M680I, V726A). One of our objective was to test and compare these two groups for the diagnostic utility of 2 adults and 1 pediatric FMF diagnostic criteria (Tel Hashomer, Livneh and Pediatric) (2-4).

Methods: The medical records of 1685 children diagnosed and followed up as FMF were reviewed retrospectively. The demographic, clinical and genetic data of children carrying only E148Q variant (either heterozygous or homozygous carriers) (group 1) and children with pathogenic homozygous mutations (M694V, M694l, M680I, V726A) (group 2) were collected. All patients were evaluated for three diagnostic criteria.

Results: Children with E148Q variant were 128 and children with pathogenic homozygous variants were 429 . Male to female ratio was 1.13 in group 1 and 1.15 in group 2 ( $>0.05)$. The mean age of patients in group 1 was $12.1 \pm 4.7$, in group was $214 \pm 5.9(p<0.001)$. The mean age of symptom onset was $5.4 \pm 4.1$ in group $1,4.3 \pm 3.6$ in group $2(p=0.01$. Consanguinity was significantly higher in group $2(29 \%$ vs $53 \%, p<0.01)$. In group $117.9 \%$ had a sibling with FMF, while in group $227 \%$ had a sibling with FMF $(p=0.03)$. Clinical features like pleuritis, synovitis, recurrent fever, erysipelas-like erythema and monoarthrtis were significantly more common in group 2 than group $1(31.7 \%$ vs $9.3 \% ; p<0.001,55.9 \%$ vs $32 \% ; \mathrm{p}<0.001,86.9 \%$ vs $78.9 \% ; \mathrm{p}<0.03,31.2 \%$ vs $7 \% ; \mathrm{p}<0.001$, $51.7 \%$ vs $28.1 \% ; p<0.001$, respectively). Incomplete attacks were significantly more common in group 1 than group $2(16.4 \%$ vs $7.4 \%$; $\mathrm{p}<0.005)$. Moderate and severe PRAS scores were significantly higher in group 2 (93.9\% vs $71.9 \% ; p<0.001)$. Laboratory values like $\mathrm{C}$-reactive protein, erythrocyte sedimentation rate, serum amyloid $A$ were found significantly higher in group 2 than group 1. (3.6 \pm 6.3 vs $0.8 \pm 1.2 ; p<0.001$, $13 \pm 10$ vs $7.3 \pm 6 ; \mathrm{p}<0.001,6.9 \pm 22.5$ vs $2.9 \pm 2.2 ; \mathrm{p}=0.007$, respectively). The percentage of children diagnosed according two Tel Hashomer and pediatric criteria was significantly higher in group 2 than group $1(88.5 \%$ vs $77.3 \% ; \mathrm{p}=0.002,95.3 \%$ vs $84.3 \% ; \mathrm{p}<0.001$, respectively). But both groups show similar diagnostic utility according to Livneh criteria.

Conclusion: Although children carrying $E 148 Q$ variants meet the three validated diagnostic criteria of FMF. They had milder course of disease than children homozygous for pathogenic MEFV variants both clinically and in laboratory means.

\section{REFERENCES}

[1] Touitou I (2001) The spectrum of Familial Mediterranean Fever (FMF) mutations. Eur J Hum Genet 9, 473-83

[2] Sohar E, Gafni J, Pras M, Heller H (1967) Familial Mediterranean fever. A survey of 470 cases and review of the literature. The American journal of medicine 43:227-253

[3] Livneh A, Langevitz P, Zemer D, Zaks N, Kees S, Lidar T, Migdal A, Padeh S, Pras M (1997) Criteria for the diagnosis of familial Mediterranean fever. Arthritis and rheumatism 40:1879-1885.

[4] Yalcinkaya F, Ozen S, Ozcakar ZB, Aktay N, Cakar N, Duzova A, Kasapcopur O, Elhan AH, Doganay B, Ekim M, Kara N, Uncu N, Bakkaloglu A (2009) A new set of criteria for the diagnosis of familial Mediterranean fever in childhood. Rheumatology (Oxford, England) 48:395-398.

Disclosure of Interests: None declared

DOI: 10.1136/annrheumdis-2019-eular.7098

\section{SAT0523 EVALUATION OF COEXISTING DISEASES IN CHILDREN WITH FAMILIAL MEDITERRANEAN FEVER}

Mehmet Yildiz, Amra Adrovic, Emre Tasdemir, Khanim Baba-Zada,

Muhammed Aydin, Oya Koker, Sezgin Sahin, Kenan Barut, Ozgur Kasapcopur. Cerrahpasa Medical School, Istanbul University Cerrahpasa, Department of Pediatric Rheumatology, Istanbul, Turkey

Background: Familial Mediterranean Fever (FMF) is most common periodic fever syndrome in childhood. It is characterized by fever attacks, abdominal pain lasting between $6-72$ hours, serositis and erysipelas like erythema. Since FMF is inherited in autosomal recessive manner, it has higher frequency in populations that have higher rate of consanguineous marriages. As it is a lifelong chronic disorder, it is important to understand its clinical course for preventive medicine. Despite the higher incidence of variety of disorders shown studies among adult FMF patient, there is not enough data from pediatric populations.

Objectives: The objective of the study is to evaluate the comorbid disorders in a large pediatric familial Mediterranean fever cohort.

Methods: Six hundred and eighty-six children with FMF were interviewed by the same investigator between October 2018 and January 2019 in our pediatric rheumatology department. Demographic features and MEFV gene mutations were recorded from patients' charts. Patients and/or their parents were asked about characteristics of their fever episodes, presence of arthralgia, arthritis, abdominal pain, chest pain in the course of fever attack and coexistence of any other disease confirmed by a physician.

Results: Female-male ratio of our cohort was 0,85 . Mean age of the patients was 12,8 $\pm 7,4$ years. Mean age of patients at disease onset and at the time of diagnosis was 4,38 \pm 3,46 years and 6,55 $\pm 3,67$ years, respectively. Sixty-five and a half percent of patients had family history of FMF. Consanguineous marriage rate was $29,8 \%$. Most common MEFV mutations were: M694V homozygotes (18,8\%), M694V heterozygotes $(17,7 \%)$, R202Q heterozygotes $(13,1 \%)$, R202Q homozygotes $(5,5 \%)$ and V726A heterozygotes $(4,9 \%)$, respectively. Resistance to colchicine treatment was present in $43(6,2 \%)$ patients. Number of patients that underwent tonsillectomy were $9(1,3 \%)$ and number of patients that had appendectomy was $11(1,6 \%)$. Detected coexisting diseases are listed in Table 1.

Detected coexisting diseases in patients group.

\begin{tabular}{lc}
\hline & $\mathrm{n}(\%)$ \\
\hline Juvenile Idiopathic Arthritis & $40(5,8)$ \\
- Systemic & $4(0,5)$ \\
- Oligoarticular & $17(2,4)$ \\
- Polyarticular & $5(0,7)$ \\
- Enthesitis Related Arthritis & $1(0,1)$ \\
- Juvenile Spondylitis & $13(1,8)$ \\
Asthma/Reactive Airway Disease & $26(3,7)$ \\
Henoch Schonlein Purpura & $18(2,6)$ \\
Uveitis & $10(1,4)$ \\
Inflammatory bowel disease & $9(1,3)$ \\
Periodic fever, aphthous stomatitis, pharyngitis, cervical adenitis (PFAPA) & $7(1)$ \\
Acute Rheumatic Fever & $7(1)$ \\
Migraine & $7(1)$ \\
Allergic Rhinitis & $6(0,8)$ \\
\hline
\end{tabular}

Conclusion: In this study, we have reported increased frequency of juvenile idiopathic arthritis, asthma- reactive airway disease, Henoch Schonlein purpura, uveitis, inflammatory bowel disease, PFAPA syndrome and acute rheumatic fever in a large pediatric FMF cohort. Those findings consistent with data from literature. It is important to be alert about these diseases that may develop in patients with FMF for preventing them from the potential morbidities.

\section{REFERENCES}

[1] Barut K, Sahin S, Adrovic A, et al. (2018). Familial Mediterranean fever in childhood: a single-center experience. Rheumatology international, 38(1), 67-74.

[2] Beser ÖF, Kasapçopur Ö, Çokugras F Ç, et al. (2013). Association of inflammatory bowel disease with familial Mediterranean fever in Turkish 